\title{
Téoros
}

Revue de recherche en tourisme

\section{Le congé solidaire ou l'utilité du voyage}

\section{Melina Schoenborn}

Volume 26, numéro 3, automne 2007

Tourisme et solidarité

URI : https://id.erudit.org/iderudit/1071007ar

DOI : https://doi.org/10.7202/1071007ar

Aller au sommaire du numéro

Éditeur(s)

Université du Québec à Montréal

ISSN

0712-8657 (imprimé)

1923-2705 (numérique)

Découvrir la revue

Citer cet article

Schoenborn, M. (2007). Le congé solidaire ou l'utilité du voyage. Téoros, 26(3),

51-55. https://doi.org/10.7202/1071007ar d'utilisation que vous pouvez consulter en ligne.

https://apropos.erudit.org/fr/usagers/politique-dutilisation/ 


\section{Le congé solidaire ou I'utilité du voyage ${ }^{1}$}

\section{Melina Schoenborn}

"Association de jeunes couturiers, Bamako, Mali, cherche comptable. Durée de l'emploi : deux semaines. Salaire: nul. Bénéfices: énormes sur le plan personnel. Prière de convaincre votre employeur actuel de payer pour votre déplacement et vos dépenses. "

Utopique comme offre d'emploi ? Pas tant que cela. Le voyage solidaire a la cote et a su se frayer un chemin jusqu'aux services des ressources humaines des grandes entreprises privées et publiques. Certaines vont même jusqu'à encourager leurs employés à devenir coopérants volontaires pendant leurs vacances annuelles, financement compris. Et plus d'un répondent à l'appel.

Étonnant ? Déjà, au milieu du siècle dernier, l'exploratrice Ella Maillart (1988 : 309) écrivait, dans un de ses récits de voyage en Asie: " Je sais, d'expérience, que courir le monde ne sert qu'à tuer le temps. On revient aussi insatisfait qu'on est parti. II faut faire quelque chose de plus. " Cette conception du voyage semble avoir trouvé un écho auprès de plusieurs touristes dans une époque où les complexes hôteliers gérés par des entreprises du Nord poussent comme des champignons. La définition même du terme tourisme semble réductrice quand on regarde les différentes formules de voyage qui s'offrent aux voyageurs actuels: vacances équitables, tourisme responsable ou solidaire. Le simple voyage d'agrément ne semble plus suffire à certains citoyens du Nord mal à l'aise devant la répartition inégale des recettes issues des activités touristiques dans les pays en développement. Où va l'argent qu'ils dépensent? Les communautés locales en profitent-elles? Selon le Bureau

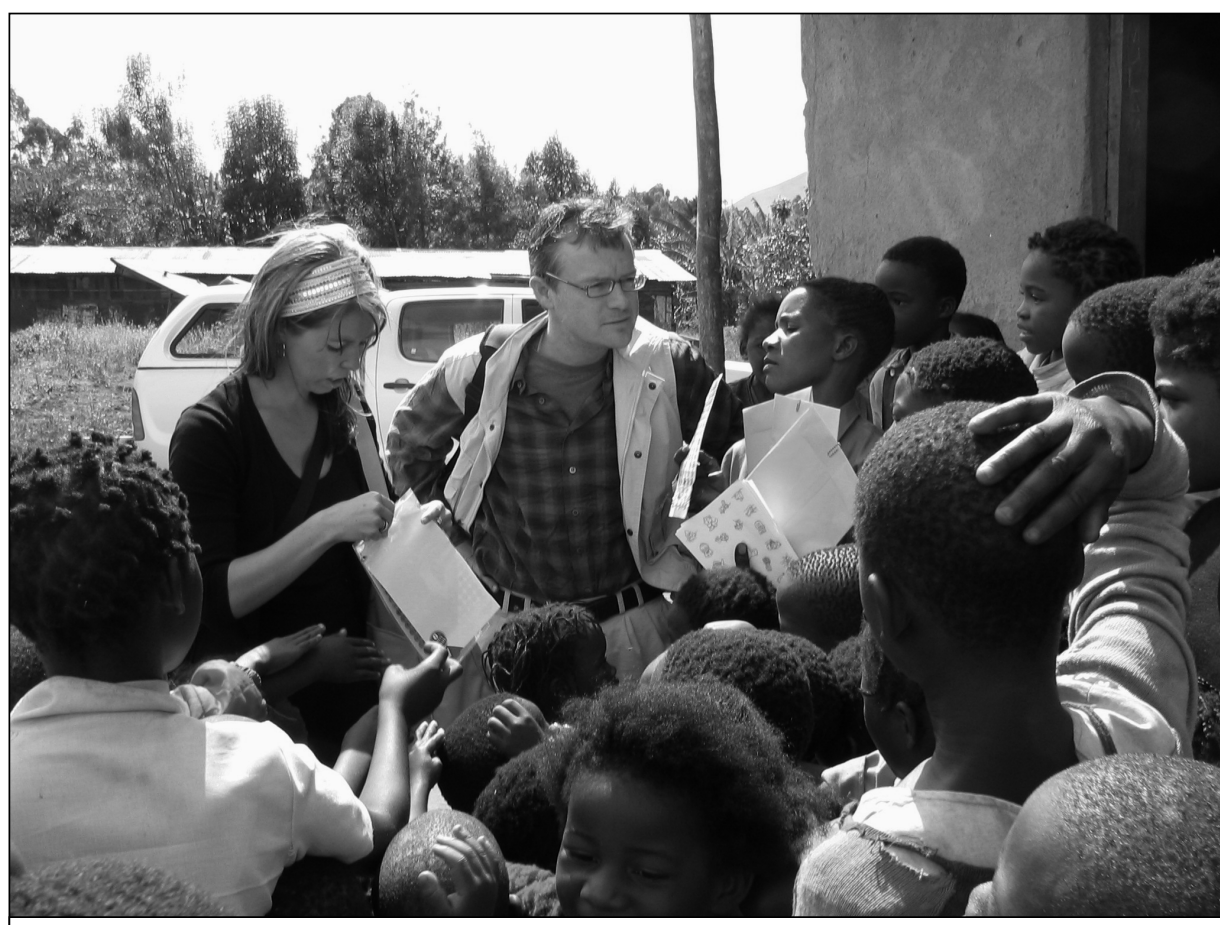

Tiffany Stone et Matthew Steele des Laboratoires Abott lors d'une campagne de sensibilisation contre le VIH/sida au Malawi.

Photo: Sylvain Matte

international du tourisme social (BITS), une part croissante des voyageurs (5 à $10 \%$ selon les pays) recherchent des séjours "plus éthiques» (Rasera, 2002-2003: 5). Auparavant spectateurs, les nouveaux touristes veulent devenir des acteurs dans les pays qu'ils visitent, consommer des produits locaux, découvrir l'Autre. Certain veulent même aller plus loin et participer aux efforts de développement dans les pays pauvres du Sud. L'engagement et l'ouverture sont à l'ordre du jour, tandis que le voyage comme mouvement de fuite, d'oubli de soi et du monde réel, comme célébration d'un hédonisme insouciant, semble ne plus convenir à tous. Résultat: de plus en plus d'agences de voyages et d'organismes de coopération internationale offrent des façons différentes de voyager et de découvrir le monde. Les termes coopération, solidarité et bénévolat font maintenant partie du voyage! Non seulement les citoyens du Nord souhaitent-ils participer aux efforts pour un monde plus équitable, mais leurs employeurs sont souvent de la partie.

Au Québec, plusieurs agences ont mis sur pied des séjours « découverte" qui répondent aux principes du tourisme équitable: les retombées financières des activités touristiques reviennent directement aux populations visitées et contribuent à leur développement socioéconomique; les options touristiques proposées privilégient les services offerts et gérés collectivement par 
les communautés locales; les voyageurs échangent avec la population du pays visité ; les voyageurs respectent les cultures locales et l'environnement. Parmi elles, l'agence Mercure ${ }^{2}$ offre des forfaits au Mexique dans lesquels les visiteurs rencontrent une organisation communautaire engagée dans l'agriculture biologique, font des activités écotouristiques offertes par un regroupement autochtone, participent aux activités d'une communauté qui se voue à la protection d'espèces en péril et achètent leurs souvenirs directement des artisans.

Le tourisme solidaire, quant à lui, implique une participation directe du voyageur dans un projet de développement local déterminé par la population d'accueil.

Horizon Cosmopolite ${ }^{3}$, entreprise québécoise fondée en 1997, offre à sa clientèle une quarantaine de stages individuels dans 25 pays d'Asie, d'Amérique latine, d'Europe et d'Afrique. Les participants doivent s'engager à travailler au sein d'une communauté pour une période minimale d'une semaine. Au programme : l'enseignement de l'anglais au Népal, le travail dans une pouponnière au Sénégal ou encore dans une coopérative de femmes artisanes en Équateur.

Habitat pour l'humanité Canada 4 propose de son coté des séjours à l'étranger (cette année au Ghana) de trois semaines pendant lesquels les participants aident à la construction d'une maison destinée à une famille à faible revenu.

\section{Entreprises et bénévoles demandés}

Pour répondre à ce souhait d'engagement, l'association Planète Urgence (France) ${ }^{5}$ a mobilisé depuis cinq ans plus de 1800 citoyens bénévoles (aussi nommé volontaires) dans des missions de développement d'une durée de deux semaines, en Afrique et en Asie. Ces missions sont destinées à des professionnels de divers domaines, y compris l'administration et la gestion, l'informatique et la bureautique ou la protection de l'environnement. Comme mesure incitative, $66 \%$ des frais encourus par la mission sont déductibles du revenu imposable pour le participant qui voyage par ses propres moyens. Sinon, le candidat peut solliciter l'aide de son employeur à l'intérieur de la formule " congé solidaire ». À ce jour, une centaine d'entreprises, dont L'Oréal, La Poste et IBM France, ont accepté de financer la totalité du coût des missions de 800 employés, par le biais de ce nouveau type de coopération internationale. L'objectif est de permettre une nouvelle forme de coopération Nord-Sud dans laquelle les compétences existantes dans les entreprises du Nord peuvent répondre aux besoins des communautés du Sud. L'entreprise privée, peu présente dans les systèmes de coopération classiques, est mise de l'avant et devient un acteur central dans la lutte contre les inégalités et la pauvreté ${ }^{\text {. }}$

Le congé solidaire est maintenant disponible au Canada grâce à une entente entre Planète Urgence et deux organismes qui ont une longue feuille de route en matière de coopération pour le développement: le Centre d'étude et de coopération internationale (CECl), basé à Montréal, et l'Entraide universitaire mondiale du Canada (EUMC), dont le siège est à Ottawa. Ces deux organismes ont mis sur pied Uniterra, le plus important programme de développement international reposant sur le bénévolat et l'engagement volontaire de citoyens et d'organisations (y compris des entreprises privées) au Canada. Uniterra intervient dans 13 pays d'Afrique, d'Asie et d'Amérique latine pour réduire la pauvreté. Les volontaires du congé solidaire deviennent donc des participants à cet important programme mis en œuvre grâce à un appui financier de l'Agence canadienne de développement international (ACDI).

"La formule du congé solidaire répondait exactement à l'un de nos objectifs du programme Uniterra : faire participer aux efforts de coopération des gens et des organisations qui n'y trouvaient pas leur compte dans les programmes existants", mentionne Sylvain Matte, directeur d'Uniterra. Normalement, les volontaires doivent quitter travail, famille et amis pour une période de un à deux ans. Or, ce ne sont pas tous les citoyens et les citoyennes qui sont prêts à partir à l'étranger pour une période aussi longue. Le congé solidaire est donc un moyen, par la coopération internationale de courte durée, de rejoindre une clientèle élargie : celle qui veut effectuer un travail humanitaire lié à sa profession pendant une partie de ses vacances annuelles.

La durée de l'affectation à l'étranger est de deux ou trois semaines. Pour les entreprises, c'est aussi une façon concrète de participer aux efforts de développement et d'exercer leur responsabilité sociale.
Le concept est alléchant : l'employeur qui devient partenaire du programme propose à ses employés de partir sur son temps de vacances ou de prendre un congé sans traitement, ou encore d'utiliser sa banque de temps accumulé. Certains offrent même une semaine de vacances de plus, ce qui donne au participant davantage de temps et de flexibilité pour organiser et réaliser son projet. Par ailleurs, l'employeur libère son employé quelques jours pour qu'il puisse suivre une formation préalable au départ, donnée par Uniterra, dans le but de le préparer aux enjeux de la coopération internationale, étant donné que plusieurs participants au programme en sont à leur première expérience de travail à l'étranger. Mais, pour chaque employé en partance pour la Bolivie, le Botswana, le Sri Lanka ou un autre des treize pays où œuvre Uniterra, l'employeur s'engage à verser une contribution financière pour couvrir les frais de déplacement, de vaccins et de logement.

L'affectation est organisée de concert avec le partenaire local dans le pays d'accueil. "Nos partenaires locaux nous informent de leurs besoins. Ensuite, nous contactons nos entreprises partenaires canadiennes et leur fournissons les offres de recrutement. Elles se chargent de passer le mot à leurs employés ", explique le directeur d'Uniterra.

\section{Pas des vacances! Mais du tourisme?}

Si le terme vacances désigne normalement une période pendant laquelle une personne cesse ses activités professionnelles pour aller faire du tourisme, le congé solidaire, lui, est tout sauf des vacances à proprement parler. "Le congé solidaire est basé sur la volonté de la personne, son désir de faire avancer les choses, de participer à la lutte contre la pauvreté en partageant ses connaissances et ses savoirs. Les gens qui s'inscrivent au programme sont choqués par la pauvreté dans le monde, ils veulent faire quelque chose, être utiles! ", ajoute S. Matte. Uniterra travaille concrètement au développement des compétences des gens qui œuvrent dans des organisations locales de lutte contre le $\mathrm{VIH} /$ sida, d'alphabétisation des femmes, des centres de santé, des écoles et de nombreuses entreprises de type coopératif ou associatif. "Avec l'appui de nos 
volontaires, les organisations développent de meilleurs services aux populations, c'est ça le développement ", nous dit Sylvain Matte. Les besoins sont donc très larges: les technologies de l'information, la vente et la mise en marché, la gestion d'épargne et de crédit, l'agroalimentaire, la création d'entreprises, les communications, la formation professionnelle et l'environnement. Le programme appuie même des initiatives de tourisme communautaire au Guatemala, au Botswana et bientôt en Bolivie. S. Matte ajoute: "Les candidats sont retenus sur la base de leur expertise. Le programme est co-financé par l'Agence canadienne de développement international (ACDI). Ce sont des fonds publics, il faut que les affectations à l'étranger apportent un plus, que le travail soit précis et qu'il y ait un partage du savoir. " À son arrivée, la personne en congé solidaire rejoint les volontaires d'Uniterra déjà sur place dans une affectation longue durée. Les congés solidaires viennent s'intégrer dans des projets à long terme. Parfois deux semaines ne suffisent pas pour terminer un projet. Dans un tel cas, un deuxième volontaire du congé solidaire prendra la relève et rejoindra à son tour l'équipe locale pour poursuivre le travail. Par exemple, un premier mandat peut servir à mettre sur pied une page Web pour une entreprise de beurre de karité au Burkina Faso, tandis que le mandat suivant servira à montrer comment mettre cette page Web à jour et faire des modifications. Au bout du compte, cela aidera les femmes productrices de karité à vendre leurs produits sur le marché international. C'est l'une des dizaines d'activités du programme Uniterra qui a conduit à accroître significativement les revenus de quelques milliers de femmes productrices de karité au Burkina Faso, au Mali, en Guinée et au Niger. Voilà des résultats concrets de développement.

À la question: le congé solidaire est-il néanmoins une forme de tourisme? S. Matte répond prudemment :

Nous tentons de nous démarquer du concept de tourisme solidaire pour parler plutôt de nouvelles tendances en coopération internationale. Le milieu de la coopération internationale est vu par plusieurs comme un milieu où seul un petit groupe d'initiés est apte à agir. Le congé solidaire révolutionne et " démocratise » la coopération pour le développement en ouvrant des possibilités à Pour nous, la notion de tourisme est liée à celle de la consommation. Les touristes voyagent d'abord pour leur plaisir, pour satisfaire leurs besoins de curiosité, de découverte, d'aventure... Le centre d'attraction est le touriste et tout est centré sur lui. Dans notre cas le centre d'attraction est le besoin de nos partenaires dans les pays pauvres, la perspective est très différente. Nous ne voulons pas que les personnes qui viennent en congé solidaire agissent comme des consommateurs qui seront plus ou moins satisfaits du service qu'on leur offre tout comme ils le feraient en évaluant leur agence de voyage. Bien entendu, les objectifs personnels des participants (découverte de nouveaux pays, de nouvelles cultures, valorisation personnelle liée au sentiment d'être utile) ne sont pas contradictoires avec ceux de la coopération pour le développement, bien au contraire. Le congé solidaire est un savoureux mélange des deux dimensions.

Le congé solidaire se veut un pas (bien modeste!) vers la réussite des objectifs du Millénaire pour le développement (OMD). Ces objectifs, une initiative de la commu- un plus grand nombre de personnes.

nauté internationale sous la direction des Nations Unies, se présentent comme des mesures concrètes visant à réduire la pauvreté de moitié dans le monde d'ici 2015.

Agnès van't Bosch, responsable du congé solidaire pour Uniterra, conclut :

Nos secteurs d'intervention sont en ligne avec les OMD, qui sont de lutter contre la faim, la maladie, l'analphabétisme, la dégradation de l'environnement et la discrimination envers les femmes. Les mandats réalisés dans le cadre du congé solidaire sont des appuis ponctuels qui s'inscrivent dans quelque chose de plus grand. C'est une contribution très personnelle aux efforts internationaux pour la réduction de la pauvreté.

Les retombées doivent être concrètes et mesurables. En ce sens, les préoccupations du CECl et de l'EUMC rejoignent celles de Planète Urgence. Sur le site Web de l'ONG (organisation non gouvernementale) française, on peut lire: "Nos missions répondent à des besoins urgents et précis. Ce ne sont pas des grands projets issus de notre vision de gens du Nord ${ }^{7}$. En effet, les trois organismes semblent vouloir se distancier de la formule «grandes œuvres de charité ».

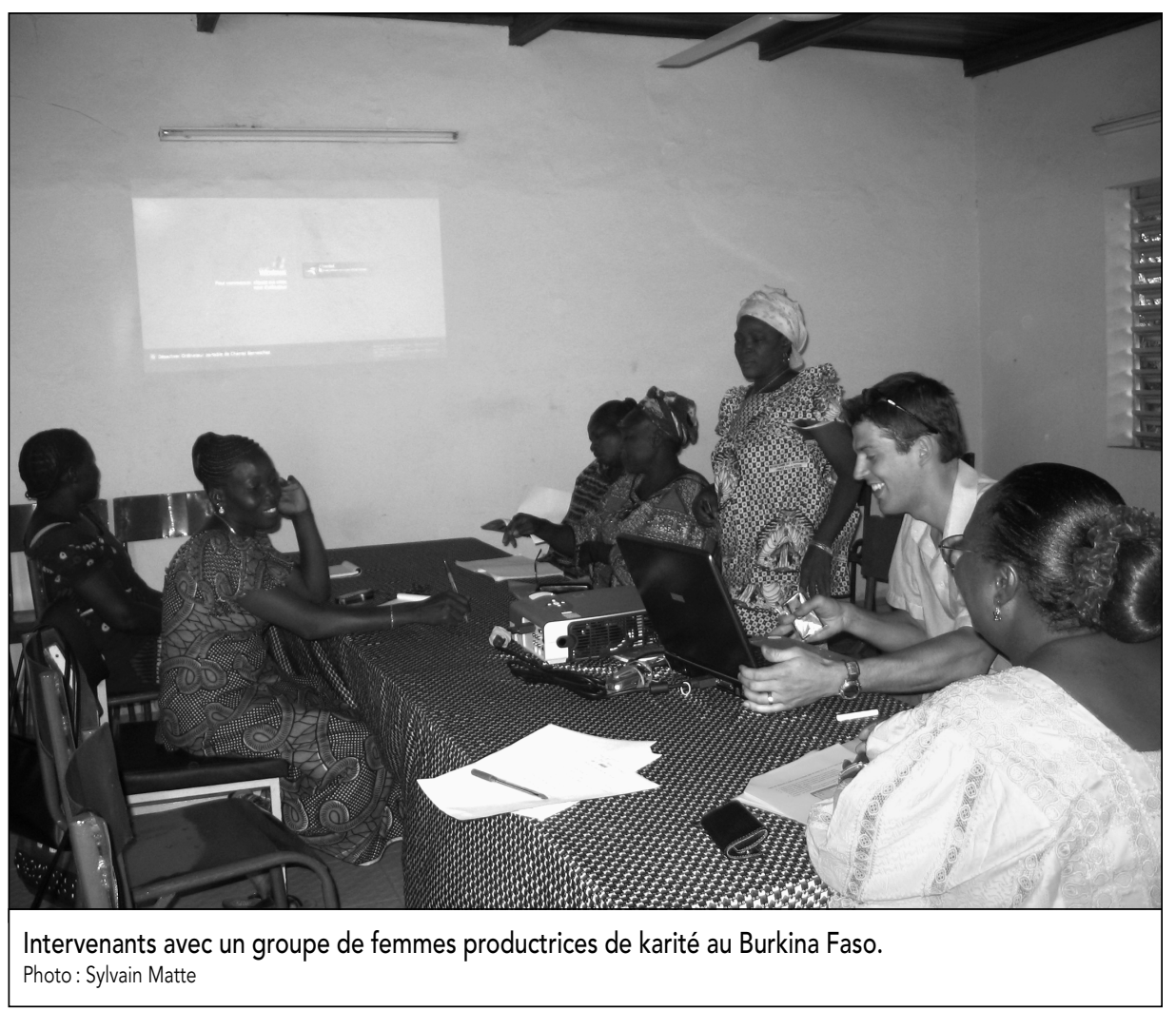


S. Matte hésite d'ailleurs à utiliser le mot mission et lui préfère le terme "affectation bénévole " quand vient le temps de décrire la nature du congé solidaire.

Les postes de congé solidaire peuvent en rebuter certains qui ont une vision plus romantique de la coopération internationale. Planète Urgence précise : «II faut demeurer réaliste et il est malheureusement impossible de 'sauver' le monde, non seulement dans une période aussi courte, mais également en essayant d'importer sur le terrain nos façons de faire pour les reproduire dans une société où tout est si différent ${ }^{8}$. " Éponger le front d'un enfant malade est un geste rempli de compassion, mais ce n'est pas nécessairement le type d'aide dont le pays d'accueil a besoin actuellement. La question n'est pas tellement: que voudrais-je faire pour améliorer le sort de la planète, mais bien : en quoi mes compétences et mes savoirs peuvent-ils être utiles au développement des capacités locales ? « II y a parfois une tension entre ce qui peut nous attirer comme personne (intérêts, motivations) versus les besoins concrets des communautés pour mener à bien leurs objectifs de développement. La bonne volonté ne suffit pas dans ce cas. Une certaine dose d'abnégation est nécessaire ", croit S. Matte. II ajoute:

certaines personnes nous ont contactés pour aller s'occuper des enfants orphelins du sida, ou pour accompagner des malades en phase terminale. C'est un très beau geste de solidarité, mais cela ne correspond pas à ce que nous tentons de faire : travailler sur les causes des problèmes de développement et aider les intervenants locaux à y remédier. II y a d'autres organisations qui offrent ce type d'expérience de solidarité à l'étranger, pas nous.

\section{Mais un retour considérable sur l'investissement !}

Depuis le lancement du congé solidaire au Québec en 2005, Uniterra a mobilisé 30 volontaires par le biais de cette formule. Selon A. van't Bosch, toutes les affectations ont été un succès. "Un mandat de deux ou trois semaines, c'est trop court pour agir ", disaient certains sceptiques. Pourtant, les volontaires du congé solidaire et les partenaires sur le terrain témoignent du contraire. Malgré la chaleur, les risques en matiè- re de santé et l'adaptation qui représentent autant de défis pour les volontaires, tous les participants ont terminé leur mandat. II leur faut beaucoup de flexibilité et d'ouverture aux changements. Les plans ne se passent pas toujours comme prévu et les méthodes de travail et d'apprentissage diffèrent souvent. Michel Arsenault, un technicien en développement d'application bureautique à la Ville de Montréal, partenaire du congé solidaire, est parti à Dakar, au Sénégal, partager ses connaissances en informatique avec 18 étudiants, majoritairement des femmes de 22 à 60 ans en processus d'alphabétisation. II témoigne de son expérience à Dakar :

Malgré le peu de moyens didactiques à ma disposition, j'ai réussi à enseigner à chacun de mes trois groupes des fonctions de base dans Word, Excel et Outlook et je trouve les résultats des plus encourageants. Ces femmes se servent de ces outils informatiques quotidiennement dans la rédaction de leur correspondance, mais aussi dans la saisie de tout autre document. Elles ont la volonté d'améliorer leurs conditions de vie et celles de leurs proches. Certaines de mes élèves continuent à m'écrire par Internet, qui leur apporte une plus grande ouverture sur le monde et surtout un accès à plus d'information. Ma vision de la vie est changée: c'est un peu comme la publicité du 6/49, c'est-àdire que devenir gagnant, cela ne change pas le monde, SAUF QUE... nos valeurs s'humanisent encore plus et on relativise plus facilement, on fait plus avec moins de moyens en faisant plus largement appel à notre créativité.

Amélie Régisse, une autre participante de la Ville de Montréal, a fait un travail fort apprécié de son partenaire local en matière de communication. "Sans l'appui d'Amélie en communication", nous confie Abdou Salam Fall du Réseau international de promotion de l'économie sociale solidaire (RIPESS), «l'organisation de notre événement

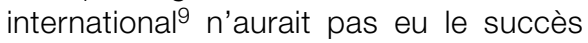
qu'il a eu ».

Ces deux employés ont participé au congé solidaire dans le contexte d'un projet pilote de deux semaines en 2005 qui visait à déterminer si, pour la Ville de Montréal, le projet était viable ou non. Avant de devenir officiellement partenaire du congé solidaire, la Ville voulait savoir si l'impact était réel sur les communautés du Sud, étant donné la courte durée du mandat et si, compte tenu des longues heures de vol, de la médication à prendre et de l'adaptation socioculturelle exigée des volontaires, le projet en valait la peine. Or, les deux participants sont revenus très enthousiastes.

" À mon retour, des gens à la Ville de Montréal ont été étonnés de mon engouement », confie M. Arsenault :

À l'évaluation de mon stage, j'ai donné $100 \%$ à l'expérience et $100 \%$ à la relation avec les Sénégalais! Et je suis sincère, croyez-moi. J'ai rencontré des gens extraordinaires. J'ai développé une belle relation avec les apprenants. [...] Une des partenaires coopérantes québécoises qui réside là-bas désire les regrouper pour qu'ils se rencontrent périodiquement. On pourra alors continuer la formation entre nous, grâce à Internet.

Quant à A. Régisse, elle affirme :

Depuis mon retour, on me demande souvent ce qui m'a le plus frappée dans cette aventure. Je pourrais répondre que la pauvreté m'a attristée, que la pollution m'a étouffée, que les coquerelles géantes m'ont traumatisée. Mais j'opterais plutôt pour louanger la générosité du peuple sénégalais, les valeurs d'entraide et de partage qui sont omniprésentes chez eux, le temps qu'ils s'accordent entre eux, le temps qu'ils accordent aux autres, la débrouillardise dont ils font preuve, leur positivisme.

Depuis, la Ville a décidé de devenir un partenaire de congé solidaire. Cinq autres volontaires sont partis en 2006 pour mener à bien des mandats au Mali, en Guinée Conakry et au Niger et le comité exécutif de la Ville de Montréal a approuvé l'organisation de cinq autres missions en 2007.

\section{Quels bénéfices pour les employeurs?}

D'autres employeurs québécois ont répondu à l'appel d'Uniterra et ont accepté de devenir partenaires du congé solidaire, dont Samson Bélair/Deloitte \& Touche, la Coopérative fédérée et la Confédération des syndicats nationaux (CSN), cette dernière en collaboration avec Équiterre. Une des raisons principales de leur engagement 
est la motivation des employés. Pendant leur séjour à l'étranger, ces derniers doivent faire preuve de courage et d'empathie, montrer de l'initiative, être responsables et prendre des décisions. Sur le terrain, ils travaillent de pair avec des gens débrouillards qui sont capables de faire beaucoup avec peu. En somme, les employés reçoivent autant qu'ils donnent; ils ramènent ces nouveaux acquis avec eux au sein de l'entreprise. "Au retour, ils essaient d'appliquer ce qu'ils ont appris là-bas ", affirme A. van't Bosch, ajoutant: "Dans l'avenir, les entreprises vont de plus en plus faire face à des difficultés de rétention de leur personnel. Ceux-ci vont choisir leur emploi non seulement sur la base du salaire et des conditions de travail, mais aussi sur la base de critères liés à la responsabilité sociale de leur employeur. » Congé solidaire donne donc aux entreprises un moyen concret d'améliorer leur image corporative interne auprès de leurs employés, tout en offrant des occasions qui seront vues comme très intéressantes pour ces derniers. "Je suis fier de travailler pour une entreprise qui a du cœur et des valeurs ", nous dit Philippe Chevalier, employé chez Samson Belair/Deloitte \& Touche, l'un des tout premiers participants au Congé solidaire. "Tout le monde y trouve son compte. Le programme s'inscrit bien dans les objectifs de la Ville de Montréal de contribuer au développement international », affirme M. Dominique, chargé du dossier Congé solidaire à la Ville de Montréal.

Sara St-Laurent, du service des ressources humaines chez Samson Belair/Deloitte Touche, explique que congé solidaire est une façon efficace d'augmenter le sentiment d'appartenance chez leurs employés :

Chaque année, la firme envoie un courriel d'introduction à ses employés pour les inviter à prendre connaissance des possibilités de congé solidaire. Beaucoup viennent me voir pour en savoir plus sur le programme. Une quinzaine de personnes s'inscrivent annuellement au processus de sélection. Nous retenons deux candidatures chaque année. À leur retour, les participants sont encouragés à partager leurs expériences avec leurs collègues. Des dîners-causeries sont organisés et les témoignages des participants figurent sur le portail interne de l'entreprise.
Le projet devient ainsi vivant dans toute l'entreprise et, à travers la contribution de quelques collègues, c'est toute une équipe d'employés qui se sent concernée.

Le volet sensibilisation du public est un autre aspect important du congé solidaire. Les gens qui travaillent constituent un public qui n'est pas visé spécifiquement par les organismes qui tentent de promouvoir la solidarité internationale. "Très souvent on prêche auprès des convertis ou dans les écoles. Nous avons choisi un autre groupe cible et le congé solidaire est un outil efficace pour rejoindre des gens dans des entreprises qui ne le seraient pas autrement ", dit Sylvain Matte.

Planète Urgence ${ }^{10}$ précise, à propos des entreprises qui ont déjà participé à leurs actions, que leur engagement est plus qu'une stratégie de communication : "Il s'agit d'une volonté de s'insérer dans un nouvel environnement sociétal, d'être partie prenante d'une société civile, de fidéliser les compétences des salariés autour de valeurs économiques, porteuses de sens". Cela dit, les entreprises bénéficient quand même d'une visibilité accrue auprès du public. À leur retour au pays, les participants du programme Congé solidaire s'engagent à témoigner de leur expérience auprès du public. Certains sont conviés à la Journée internationale des volontaires (le 5 décembre), d'autres organisent des levées de fonds. Les Laboratoires Abbott, dont six de leurs employés ont participé au programme, a même présenté lors d'une réunion internationale à Porto Rico un DVD sur le congé solidaire et les expériences de leurs employés.

Pour les convaincre de devenir partenaire du congé solidaire, Agnès van't Bosch fait du démarchage auprès des entreprises dans le reste du Canada:

Après les succès au Québec, nous sommes maintenant dans la phase où nous voulons élargir le bassin d'employeurs participants à travers le Canada. Nous voulons augmenter de $60 \%$ la proportion de partenaires issus du secteur privé et trouver des collaborateurs au sein des universités.
L'Université de Guelph en Ontario est aussi partenaire du congé solidaire. Uniterra a pour objectif de réaliser 100 congés solidaires cette année et tentera de développer ce concept de manière à répondre à la demande de ses partenaires du Sud selon le potentiel de l'offre canadienne qui, pour le moment, ne semble pas se tarir !

Melina Schoenborn est recherchiste et rédactrice pigiste et collabore régulièrement aux publications du Centre d'études et de coopération internationale (CECl).

\section{Notes}

1 Les propos des intervenants proviennent d'entrevues réalisées par l'auteur pour les fins de l'article. Cependant ceux de Michel Arseneault et d'Amélie Regisse sont tirés d'un rapport d'Uniterra que l'on retrouve sur le site web d'Uniterra/section Congé solidaire: [www.uniterra.ca].

2 Mercure, [www.tourisme-equitable.qc.ca].

3 Horizon Cosmpolite, [www.horizoncosmo polite.com].

4 Habitat pour l'humanité Canada, [www.habi tat.ca].

5 Planète urgence,[www. planete-urgence.org].

6 [www.planete-urgence.org/esp_presse/Dos sier_Presse_PU_MAJ_fev_2007.pdf], p. 4.

7 Ibid

8 [http://www.uniterra.ca/uniterra/fr/conge_ solidaire/recits_d_entreprises_partenaires/ samson_belair_deloitte_\&_touche__stepha nie_boucher_et_christine_bertrand.html].

$9 \mathrm{La}^{\mathrm{e}}$ rencontre sur la globalisation des solidarités, au Sénégal en novembre 2005.

10 [http://www.planete-urgence.org/esp_entre prise/concept.php].

\section{Bibliographie}

Les objectifs du Millénaire pour le développement : [www.un.org/french/millenniumgoals].

Maillart, Ella (1988), La voie cruelle, Paris, Petite bibliothèque Payot / Voyageurs.

Rasera, Carla (2002-2003), « Un 'autre' tourisme est possible, le tourisme solidaire et développement durable ", Le tourisme social dans le monde, revue trimestrielle du Bureau international du tourisme social (BITS), $n^{\circ} 140$, octobre-mars, p. 4-6. 